\title{
EXAMPLES OF HARMONIC FUNCTIONS*
}

\author{
BY A. J. MARIA
}

1. Introduction. It is known that a function $u(r, \theta)$, harmonic in the unit circle and having the property that the absolute continuity of $\int_{\theta_{1}}^{\theta_{2}}|u(r, \theta)| d \theta$ is uniform with respect to $r<1$, is uniquely determined by the boundary values which it takes on nearly everywhere, for approach merely along radii. If, however, the less restrictive condition,

$$
\int_{0}^{2 \pi}|u(r, \theta)| d \theta<M
$$

for $r<1$, is satisfied, then $u(r, \theta)$ is not necessarily uniquely determined by the boundary values which it takes on even in the wide sense nearly everywhere. $\dagger$ In fact, if infinite boundary values are allowed, two harmonic functions, $u_{1}(r, \theta)$ and $u_{2}(r, \theta)$, each having the property that the integral from 0 to $2 \pi$ with respect to $\theta$ of its absolute value is bounded for $r<1$, can take on the same boundary values everywhere in the wide sense and yet fail to be identical. Such are, for instance, the two functions

$$
\frac{a\left(1-r^{2}\right)}{1-2 r \cos \theta+r^{2}}, \frac{b\left(1-r^{2}\right)}{1-2 r \cos \theta+r^{2}}, \quad a \neq b .
$$

The harmonic function

$$
\frac{\partial}{\partial \theta} \frac{1-r^{2}}{1-2 r \cos \theta+r^{2}}
$$

takes on the value zero along all radii of the unit circle. $\ddagger$ On the other hand, a harmonic function which takes on continuously at each point of a circle a finite boundary value is uniquely determined by those boundary values. The facts are the same for three or more dimensions.

These results lead one to inquire if a harmonic function is unique when it takes on in the wide sense at each point of a

* Presented to the Society, September 10, 1931.

$\dagger$ G. C. Evans, The Logarithmic Potential, 1927, pp. 46-54.

$\ddagger$ G. C. Evans, this Bulletin, vol. 37 (1931), p. 143. 
circle or sphere, as the case may be, a given finite boundary value. It will be shown, by means of examples, that this is not necessarily true.

Another query, related to the above, concerns the Cauchy integral representation of a function, $f(z)$, analytic in the unit circle. The function $f(z)$ will be said to have a normal Cauchy integral representation if

$$
f(z)=\frac{1}{2 \pi i} \int_{c} \frac{u(t)+i v(t)}{t-z} d t,
$$

where $u(t)$ and $v(t)$ are real, summable functions on the unit circle and $f(z)$ takes on the value $u(t)+i v(t)$ in the wide sense at nearly every point of the unit circle.* The question is the following: If $f(z)$ takes on, in the wide sense, summable boundary values $u(t)+i v(t)$ nearly everywhere, is it given by (1)? It will be shown that even if $f(z)$ takes on continuous boundary values in the wide sense everywhere, it is not necessarily given by $(1) . \dagger$

2. Approach in the Wide Sense. A variable point $M$, interior to a circle or sphere, is said to approach a fixed point $P$ of a circle or sphere in the wide sense if there exists a positive constant $\alpha<\pi / 2$ such that the angle $\theta,-\pi / 2<\theta<\pi / 2$, between the ray $P M$ and the interior normal at $P$ satisfies the inequality $|\theta|<-\alpha+\pi / 2$ as $M$ approaches $P$.

A function $f(M)$ takes on a boundary value $h(P)$ at $P$ in the wide sense if $\lim f(M)=h(P)$ when $M$, interior to the circle or sphere, approaches $P$ in the wide sense. The function takes on the boundary value continuously if $\lim f(M)=h(P)$ when $M$, interior to the circle or sphere, approaches $P$ in an arbitrary manner.

3. A Function Harmonic in the Unit Circle which Approaches Zero at Every Point of the Circle in the Wide Sense and Is Not Identically Zero. The simplest case is that where approach is merely in the wide sense at just one point and continuous elsewhere.

If there is a function, $u(x, y)$, harmonic in the unit circle,

* G. C. Evans, The Logarithmic Potential, 1927, p. 66.

$\dagger$ These problems were suggested to me by Professor G. C. Evans. 
which approaches zero continuously at all points of the circle except at $P$ and approaches zero in the wide sense at $P$, then the analytic function which has this harmonic function as its real part becomes, by means of a conformal transformation which carries the interior of the unit circle into the upper half plane and the point $P$ into the infinite point, an entire function which has the property that it is pure imaginary along the real axis and that its real part approaches zero uniformly as the variable point approaches the infinite point in any sector, with vertex at the origin, whose sides are contained in the upper half plane. Conversely, the real part of an entire function having this property becomes, by means of a conformal transformation which carries the upper half plane into the interior of the unit circle, a harmonic function satisfying the given conditions.

It turns out that the entire function*

$$
f(z)=u+i v=i \sum_{2}^{\infty} \frac{z^{n-2}}{\Gamma\left(1+\frac{1}{(\log n)^{a}}\right)}, \quad(0<a<1),
$$

has this property. In fact, Malmquist obtains the inequality

$$
|f(z)|<r^{-\epsilon} M(\phi), \quad \phi=\arg z, \quad r=\bmod z,
$$

where $0<\epsilon<1$ and $M(\phi)$ may be taken as constant for all $\phi$ such that $0<\alpha<\phi<2 \pi-\alpha$. In this inequality, $\epsilon$ is independent of $a$.

4. The Three-Dimensional Case. Let us define $u_{1}(x, y)$ and $v_{1}(x, y)$ as follows: $u(x, y-1)=u_{1}(x, y)$ and $v(x, y-1)=v_{1}(x, y)$. Obviously, $u_{1}(x, y) \cdot v_{1}(x, y)$ is harmonic, since it is, except for a constant factor, the imaginary part of $\left[u_{1}(x, y)+i v_{1}(x, y)\right]^{2}$. The function $U(x, y, z)=u_{1}(x, y) \cdot v_{1}(x, y)$ is a harmonic function in three dimensions.

It now follows from these definitions and the preceding paragraph that $U(x, y, z)$ vanishes at every finite point of the plane $y=1$ and satisfies the inequality

$$
|U(x, y, z)|<\rho^{-2 \epsilon}[M(\phi)]^{2},
$$

where, in particular, $\epsilon$ may be chosen so that $1 / 2<\epsilon<1, \rho$ being the distance from $(0,1,0)$ to the projection of $(x, y, z)$ on the

* J. Malmquist, Acta Mathematica, vol. 29 (1905), pp. 203-215. 
plane $z=0$, and $\phi$ the angle which $\rho$ makes with the positive axis of $x$. We may take $M(\phi)$ as a constant for all $\phi$ such that $0<\alpha<|\phi|<\pi-\alpha$.

If an inversion is made in the unit sphere, the space $y>1$ will go into the interior $\Sigma$ of the sphere $S$ of radius $1 / 2$ and center at $(0,1 / 2,0)$. The function

$$
V(X, Y, Z)=\frac{1}{R} U\left(\frac{X}{R^{2}}, \frac{Y}{R^{2}}, \frac{Z}{R^{2}}\right),
$$

where $R=\left(X^{2}+Y^{2}+Z^{2}\right)^{1 / 2}$, and where $(X, Y, Z)$ is the point into which $(x, y, z)$ is transformed by the inversion, is harmonic in $\Sigma$. It takes on the boundary value zero continuously at each point of $S$ except $(0,0,0)$.

Consider now a point $M(X, Y, Z)$ in $\Sigma$ which approaches $(0,0,0)$ in the wide sense. Since approach is in the wide sense and the inversion is conformal, the point $(x, y, z)$ which corresponds to $M(X, Y, Z)$ will eventually lie within a right circular cone having as its axis the axis of $y$ and vertical halfangle $\phi$ which satisfies the inequality $|\phi|<\pi / 2-\alpha, \alpha$ being a positive number. The magnitude of the projection on the axis of $y$ of the line segment having as its end points $(0,1,0)$ and $(x, y, z)$ is not greater than $\rho$; the number $\rho$ has been defined following formula $\left(1^{\prime}\right)$. It then follows that

$$
r^{\prime} \sin \alpha \leqq \rho,
$$

where $r^{\prime}=\left(x^{2}+(y-1)^{2}+z^{2}\right)^{1 / 2}$. Moreover, it is evident that we have $\lim r^{\prime} / r=1$ as $r$ becomes infinite, where $r=\left(x^{2}+y^{2}+z^{2}\right)^{1 / 2}$. From these facts, one finds that

$$
\begin{aligned}
|V(X, Y, Z)|= & \frac{1}{R}|U(x, y, z)|<\frac{[M(\phi)]^{2} r}{[\sin \alpha]^{2} \gamma^{\prime} \epsilon} \\
& <\frac{2[M(\phi)]^{2}}{[\sin \alpha]^{2 \epsilon}}\left[R^{2}\right]^{\epsilon-1 / 2}
\end{aligned}
$$

for all points $M(X, Y, Z)$ which are sufficiently near $(0,0,0)$. This proves that $V(X, Y, Z)$ approaches zero in the wide sense at $(0,0,0)$.

5. Normal Cauchy Integral Representation. Consider the function 


$$
g(z)=\frac{f(z)}{i e^{f(z) / i}},
$$

where $f(z)$ has been defined in $\S 3$. If the upper half plane is transformed conformally into the interior of the unit circle, $g(z)$ will be transformed into a function $l(z)=u(z)+i v(z)$, analytic in the interior of this circle. The function $l(z)$ takes on real values continuously on the circle except at one point where it takes on in the wide sense the value zero. In fact $f(z) / i$ is real along the real axis; it approaches zero uniformly as $z$ approaches the infinite point in such a way that $\phi$, the argument of $z$, satisfies the relation $0<\alpha<\phi<2 \pi-\alpha$ (see paragraph 3 ). Hence $g(z)$ approaches zero uniformly as $z$ approaches the infinite point subject to the above condition. It is further evident, since we know that $\lim f(z) / i=+\infty$ as $z$ becomes infinite along the positive real axis, that these boundary values are continuous, and therefore, a fortiori, summable.

If $l(z)$ has a normal Cauchy integral representation, then the absolute continuity of $\int_{\theta_{1}}^{\theta_{2}}|l(z)| d \theta$ is uniform for $r<1$. $^{*}$ Since $|v(r, \theta)| \leqq|l(r, \theta)|$, it follows that the absolute continuity of $\int_{\theta_{1}}^{\theta_{2}}|v(r, \theta)| d \theta$ is uniform for $r<1$. This is a contradiction since $v(r, \theta)$ (see Introduction) would vanish identically and this would imply that $l(z)$ is a constant, which it is not.

Houston, Texas

\section{NOTE ON RELATIONS BETWEEN ELLIPTIC INTEGRALS AND SCHLÖMILCH SERIES}

BY W. R. BENNETT

In a recent study of the problem $\dagger$ of calculating modulation products in the output of a rectifier when two sinusoidal waves of different frequencies are applied, a number of interesting and apparently novel mathematical relations were deduced as a byproduct of the analysis. Unless otherwise noted, $0 \leqq b \leqq a$, $a>0, a+b \leqq \pi$, and $m$ and $n$ may be any positive integers or zero. The following theorems are typical of the results obtained.

* Fichtenholz, Fundamenta Mathematicae, vol. 13 (1929), pp. 1-33.

$\dagger$ A paper discussing this problem and its solution has been prepared for publication elsewhere. 